\title{
Pharmacological and Surgical Treatment of Intracranial Hypertension
}

\author{
Navin R. Changoor ${ }^{1}$ • Adil H. Haider ${ }^{1}$
}

Published online: 10 July 2015

(C) Springer International Publishing AG 2015

\begin{abstract}
Management of intracranial hypertension secondary to traumatic brain injury is crucial to optimizing outcomes. Use of pharmacological and/or surgical management is often needed to prevent secondary brain injury and its immediate and long-term effects. In addition to discussing medical and surgical options for approaching intracranial hypertension, this chapter will review its pathophysiology and discuss key aspects of intracranial pressure monitoring.
\end{abstract}

Keywords Traumatic brain injury · Intracranial hypertension · ICP monitoring $\cdot$ Craniectomy $\cdot$ Outcomes

\section{Introduction}

Traumatic brain injury (TBI) remains a major public health concern and is a leading cause of mortality and disability in the USA; on average, approximately 53,000 deaths per year are attributable to TBI [1]. Fall-related deaths are also on the rise, with the highest number among adult patients aged $\geq 75$ years. This highlights a new dimension of brain injury consisting of older patients whose primary mechanism of injury is a fall. These patients have diminished recovery potential, often have significant comorbidities, and may be on one or more anticoagulants [2].

This article is part of the Topical Collection on Blunt Head Trauma

Adil H. Haider

ahhaider@partners.org

1 Center for Surgery and Public Health, Brigham and Women's Hospital, 1620 Tremont Street, 4-020, Boston, MA 02120, USA
Initial or primary head injury is the initial impact that leads to disruption of brain tissue and/or blood vessels. This insult-which can be accompanied by hemorrhage, infarction, edema, or other effects of direct injury - may lead to raised intracranial pressure, as humans have an inflexible skull. Secondary brain injury refers to hypo-perfusion of brain tissue after the initial insult and is significantly associated with worse outcomes. Hypoxia, hypotension, increased intracranial pressure, and altered cellular biochemical processes may all contribute to this secondary injury. A major goal of treatment is to prevent this secondary brain injury.

Existing literature corroborates the importance of maintaining cerebral perfusion pressure despite the intracranial pressure. In all cases of TBI and resultant intracranial hypertension, a thorough understanding of the cerebral perfusion pressure is integral to preventing secondary brain injury and improving outcomes.

This chapter focuses on an overview of the relevant pathophysiology of intracranial hypertension, reviews intracranial monitoring, pharmacology, surgical options, and challenges in management, and presents a brief discussion of the prognostic indicators.

\section{Pathophysiology}

The Monro-Kellie hypothesis and cerebral dynamics are important in order to understand the pathophysiology of intracranial hypertension. The Monro-Kellie hypothesis states that the sum of the intracranial volumes of blood, brain, cerebrospinal fluid, and other components is constant and that an increase in any one of these must be offset by an equal decrease in another, or else the pressure increases [3]. The volume of these compartments is tightly regulated, and cerebral blood flow is kept constant by 
the normal regulatory process called pressure auto-regulation. Increases in intracranial pressure can result in decreases in the cerebral perfusion pressure, as shown by the following equation [2]:

cerebral perfusion pressure $(\mathrm{CPP})=$ mean arterial pressure $(\mathrm{MAP})$ - intracranial pressure $(\mathrm{ICP})$

The brain is able to maintain a normal cerebral blood flow (CBF) with a CPP that ranges from 50 to $150 \mathrm{mmHg}$ where the CPP provides the driving force behind the CBF. A reduction in $\mathrm{CBF}$ can then lead to inadequate brain tissue perfusion and oxygenation. The brain may not be able to compensate adequately at $\mathrm{CPP}$ values less than $50 \mathrm{mmHg}$ and after injury, where the ability of the brain to auto-regulate may be disrupted. The resultant brain ischemia can then further exacerbate brain edema and worsen ICP.

The surgeon must always be aware of the secondary injury processes that follow the primary injury, mainly hypotension and hypoxia. These are preventable conditions that can have a significant effect on the outcome. In fact, a single episode of hypotension can increase the morbidity of any given brain injury [4]. Therefore, an important goal in the resuscitation of these patients should be to restore the blood pressure. Hypoxia is also an independent predictor of poor outcome and must be avoided [5].

\section{Intracranial Pressure Monitoring}

Intracranial pressure monitoring is an invasive technique with associated risks and should only be utilized for patients with a depressed neurologic status (Glasgow coma scale [GCS] less than 8) or who cannot give a reliable, reproducible exam. There are several ways to monitor ICP based on the anatomical location of the monitor (Table 1). Intraventricular catheters sit in the ventricle and are considered the most accurate method to monitor ICP [6]. They are easy to recalibrate and have the additional therapeutic advantage of CSF drainage that can be helpful in intracranial lesions when edema is not the primary cause of elevated ICP. They are best used for patients who have large ventricles or where drainage would be helpful. However, it is the most invasive method and can be a challenge to insert, especially in compressed or displaced ventricles. It is also associated with infection and hemorrhage $[7,8]$. Intraparenchymal monitors are catheters that can be inserted directly into the brain tissue. Advantages include easier placement and less risk of infection and hemorrhage. However, they do not have the ability to drain CSF and cannot be recalibrated easily without the removal of the catheter. Subarachnoid bolts/screws and epidural monitors are less accurate and are less popular options. However, they have the advantages of presenting a low risk of infection and hemorrhage and can be considered in coagulopathic patients in need of ICP monitoring.

\section{Pharmacological Treatment}

Therapeutic strategies involve the use of osmotic therapy with mannitol or hypertonic saline to decrease ICP. Mannitol has been one of the first choices in the treatment of ICP due to trauma [9]. Mannitol increases the serum osmolality, resulting in the creation of an osmotic gradient that allows fluid from the cerebral parenchyma to be drawn into the serum, reducing cerebral edema and in turn ICP. While both mannitol and

Table 1 Advantages and disadvantages of ICP monitors

\begin{tabular}{|c|c|c|}
\hline & Advantages & Disadvantages \\
\hline Ventricular & $\begin{array}{l}\text { - Low cost } \\
\text { - CSF drainage } \\
\text { - Ease of recalibration }\end{array}$ & $\begin{array}{l}\text { - Most invasive } \\
\text { - Difficulties with insertion due to compression or displacement } \\
\text { of ventricles } \\
\text { - Measurement inaccuracies from blocked catheters }\end{array}$ \\
\hline Intraparenchymal & - Ease of insertion especially in those with compressed ventricles & $\begin{array}{l}\text { - Difficult to recalibrate } \\
\text { - Measurement drift over time } \\
\text { - Inability to drain CSF }\end{array}$ \\
\hline Subarachnoid & $\begin{array}{l}\text { - Ease of insertion } \\
\text { - Less invasive } \\
\text { - Lower risk of infection/hemorrhage }\end{array}$ & $\begin{array}{l}\text { - Measurement drift over time } \\
\text { - Inability to drain CSF } \\
\text { - Less accurate - often clog with debris }\end{array}$ \\
\hline Epidural & $\begin{array}{l}\text { - Ease of insertion } \\
\text { - Less invasive } \\
\text { - Lower risk of infection } \\
\text { - Lower risk of hemorrhage—-safer in coagulopathic patients }\end{array}$ & $\begin{array}{l}\text { - Less accurate - dura may dampen the pressure } \\
\text { - Measurements drift over time } \\
\text { - Inability to drain CSF }\end{array}$ \\
\hline
\end{tabular}


hypertonic saline are effective at lowering mortality, hypertonic saline seems to confer a greater survival benefit when compared to mannitol [10]. Additionally, hypertonic saline has also been shown to produce a more significant reduction in ICP when compared to mannitol [11]. While the benefits of hypertonic saline are clear, there is a lack of consensus over optimal concentration, administration route, and length of treatment. However, regardless of the concentration or administration mode (bolus or continuous), hypertonic saline has been shown to produce better short-term outcomes. The minimal adverse effects associated with hypertonic saline are yet another advantage of its use in intracranial hypertension. Furthermore, hypertonic saline has the additional advantage of having sodium level as a target and parameter to follow for effectiveness of treatment [11].

Mannitol can cause hypovolemia and hypotension and should be used with caution in the polytrauma patient, as it can cause a precipitous fall in systemic blood pressure and further exacerbate cerebral ischemia. It can also exacerbate cerebral edema if administered late after cerebral injury, due to a disrupted blood brain barrier.

Sedation also forms an important part of the pharmacological management of intracranial hypertension. These patients are often agitated, and sedation can help to reduce intracranial hypertension. However, the need for sedation must be carefully balanced against the potential loss of neurologic assessments. Propofol is one of the preferred drugs for sedation in these patients. Studies have shown favorable cerebral effects, including a reduction in ICP [12-14]. It has a relatively quick onset and offset of action, allowing for discontinuation of sedation to perform reliable clinical neurological examinations. However, one should be aware of its hemodynamic effects, as it can cause a reduction in the mean arterial pressure, which may require fluid resuscitation or even vasopressors to maintain the CPP. Propofol offers additional benefits such as increased seizure threshold and offers a better quality of sedation when compared to midazolam [15].

Barbiturates have been recommended as a sedative for patients with refractory intracranial hypertension. Literature supports its use as a mechanism to slow brain metabolism, reduce cerebral blood flow, and improve oxygenation of the cerebral tissue [16-20]. Similarly, as is the case with propofol, one should be aware of its effects on hemodynamics and be prepared to act accordingly. Loss of the neurologic exam during barbiturate coma warrants the need for continuous EEG monitoring. This requirement makes barbiturate coma unavailable at certain centers where continuous EEG monitoring is inaccessible.

In the past, ketamine was contraindicated in the management of intracranial hypertension because of adverse effects on cerebral hemodynamics. More recently, studies show that it does not worsen intracranial pressure or mean perfusion pressure [21]. Despite the absence of a therapeutic component, it has been shown to be a reasonable option for those patients with hemodynamic compromise who require sedation. Opioids and benzodiazepines have a greater role in the recovery period for long-term analgesia and sedation in the critical care setting for neurologic injury patterns.

\section{Surgical Treatment}

Decompressive craniectomy provides a larger reserve to compensate for increased pressure and circumvents the MonroeKellie doctrine, thus alleviating intracranial hypertension. Its role in the management of traumatic brain injury is not well defined and continues to be a source of debate in the literature.

Most studies have found that decompressive craniectomy is effective at reducing ICP but that there is no change in overall outcome of the patient. Studies further show that decompressive craniectomy can significantly lower ICP and may shorten the length of stay in the intensive care unit [22•]. A recent review found an associated reduction in mortality with the declining use of decompressive craniectomy in Washington State Trauma Registry but found no change in functional outcome or long-term mortality [23••]. However, Dubose et al. found that mortality was significantly better among military casualties who had higher rates of neurosurgical intervention for TBI when compared to their civilian counterparts in the National Trauma Data Bank [24]. Thus, there remains debate regarding its use. In fact, older literature, including the 2006 Cochrane review by Sahuquillo et al., found that routine use of decompressive craniectomy was not supported by the literature. Hence, clinical circumstances should be carefully considered before surgical intervention $[22 \bullet]$.

The timing of decompressive craniectomy is also debatable. Some studies suggest that early decompression lowers ICP but does not affect mortality. An evaluation of 11 level 1 trauma centers found that early decompressive craniectomy did not significantly improve mortality in patients with refractory intracranial hypertension when compared with medical therapy [25•]. In contrast, a two-center study evaluating decompressive craniectomy and lesion evacuation found that early intervention might improve outcome even when intracranial pressure was not necessarily elevated or a factor in surgical decision-making [26•].

\section{Challenges in Management}

\section{Head Injury in Intoxicated Patients}

Intoxicated patients with head injuries present a particularly challenging diagnostic and therapeutic dilemma to trauma surgeons and emergency department (ED) physicians alike. 
Alcohol intoxication is a major predisposing factor for head trauma. The management of the head-injured patient is highly dependent on an accurate clinical assessment of the patient's consciousness. The unreliability of this exam can complicate the decision-making process and prevent timely management decisions. Furthermore, these patients are agitated, disruptive, and uncooperative. In the interest of the patient and completing the clinical assessment, sedation is sometimes warranted. Benzodiazepines such as lorazepam and midazolam should be used with caution in such cases. While benzodiazepines can reduce $\mathrm{CBF}$ and ICP, there is evidence that bolus doses can significantly reduce MAP and CPP [27]. Other important disadvantages include significant respiratory depression and inhibition of the cough reflex, which can in turn predispose to aspiration.

\section{Head Injury in Multiply-Injured Patients}

Multiply-injured patients with head injuries can present a diagnostic conundrum. While the primary survey of the ATLS protocol prioritizes the rapid assessment and treatment of lifethreatening injuries, one should be aware of the effects of mannitol administration for head injuries. Mannitol can cause a deleterious drop in the blood pressure through hypovolemia and can therefore exacerbate early class I or II shock. In such cases, a fine balance is needed between resuscitation and mannitol administration.

\section{Prognostic Indicators and Rehabilitation}

The rehabilitation phase can be life-long, with functionally limiting complications that can impede therapy. There are no perfect predictors of prognosis when considering traumatic brain injury. Therapy should begin in the intensive care unit and should actively involve the patient and their family members. In certain situations, there are no therapies or medications that can substitute the support of family. We believe that family is more important than any medication or drug in improving outcomes after traumatic brain injury.

Trauma patients and their families should be made aware of the imperfect science of gauging neurologic recovery after traumatic injury. Even though prognostic calculators have been developed, based on the International Mission for Prognosis and Clinical Trial (IMPACT) database and the Corticosteroid Randomization after Significant Head Injury (CRASH) trial database, controversy still exists surrounding reliable prognostic indicators. The IMPACT group developed a numeric scale for predictors of prognosis in brain-injured patients [28], but Olivecrona et al. found that the scoring did not necessarily correlate with severe brain injury patterns [29]. The CRASH prognostic calculator was also criticized for overestimating risk of mortality and unfavorable outcomes [30]. In an effort to validate these tools in recent larger datasets, Roozenbeek et al. compared IMPACT and CRASH prognostic models; they found good generalizability and confirmed validity in quantifying prognosis. Predictors that captured most information were age, GCS score, and pupillary reactivity; no relevant differences were found between the models [31].

\section{Conclusions}

Intracranial hypertension secondary to traumatic brain injury is a significant cause of morbidity and mortality in the USA and is a major public health concern. Prevention of secondary brain injury immediately after the primary insult is essential to the successful outcomes after head trauma. Rapid development of increased intracranial pressure should be detected and treated promptly (either medically or surgically) and, whenever possible, prevented.

\section{Compliance with Ethics Guidelines}

Conflict of Interest Drs. Changoor and Haider declare no conflicts of interest.

Human and Animal Rights and Informed Consent This article does not contain any studies with human or animal subjects performed by any of the authors.

\section{References}

Papers of particular interest, published recently, have been highlighted as:

- Of importance

•- Of major importance

1. Coronado VG, Likang X, Basavaraju SV, McGuire LC, Wald MM, Faul MD, et al. Surveillance for traumatic brain injury-related deaths: United States. 1997-2007. Atlanta: US Department of Health and Human Services, Center for Disease Control and Prevention; 2011.

2. Mokri B. The Monro-Kellie hypothesis applications in CSF volume depletion. Neurology. 2001;56(12):1746-8.

3. Mulholland MW, Lillemoe KD, Doherty GM, Maier RV, Simeone DM, Upchurch GR. Greenfield's surgery: scientific principles and practice. Lippincott Williams \& Wilkins. 2012.

4. Chesnut RM, Marshall LF, Klauber MR, et al. The role of secondary brain injury in determining outcome from severe head injury. $\mathrm{J}$ Trauma. 1993;34:216-22.

5. Jones PA, Andrews PJ, Midgley S, et al. Measuring the burden of secondary insults in head-injured patients during intensive care. J Neurosurg Anesthesiol. 1994;6:4-14.

6. Adelson PD, Bratton SL, Carney NA, et al. Guidelines for the acute medical management of severe traumatic brain injury in infants, children, and adolescents: chapter 7 intracranial pressure monitoring technology. Pediatr Crit Care Med. 2003;4:S28-30. 
7. Mayhall CG, Archer NH, Lamb VA, et al. Ventriculostomy-related infections. A prospective epidemiologic study. N Engl J Med. 1984;310:553.

8. Holloway KL, Barnes T, Choi S, et al. Ventriculostomy infections: the effect of monitoring duration and catheter exchange in 584 patients. J Neurosurg. 1996;85:419.

9. Brain TF. Guidelines for the management of severe traumatic brain injury. VIII. Intracranial pressure thresholds. J Neurotrauma. 2007;24:S55.

10. Wakai A, Roberts I, Schierhout G. Mannitol for acute traumatic brain injury. Cochrane Database Syst Rev. 2007;1.

11. Mortazavi MM, Romeo AK, Deep A, Griessenauer CJ, Shoja MM, Tubbs RS, et al. Hypertonic saline for treating raised intracranial pressure: literature review with meta-analysis: a review. J Neurosurgery. 2012;116(1):210-21.

12. Flower $\mathrm{O}$, Hellings S. Sedation in traumatic brain injury. Emergency Medicine International. 2012: 11.

13. Pinaud M, Lelausque JN, Chetanneau A, Fauchoux N, Menegalli D, Souron R. Effects of propofol on cerebral hemodynamics and metabolism in patients with brain trauma. Anesthesiology. 1990;73(3):404-9.

14. Farling PA, Johnston JR, Coppel DL. Propofol infusion for sedation of patients with head injury in intensive care. A preliminary report. Anaesthesia. 1989;44(3):222-6.

15. Ronan KP, Gallagher TJ, George B, Hamby B. Comparison of propofol and midazolam for sedation in intensive care unit patients. Crit Care Med. 1995;23(2):286-93.

16. Messeter K, Nordstrom CH, Sundbarg G, et al. Cerebral hemodynamics in patients with acute severe head trauma. J Neurosurg. 1986;64:231

17. Lafferty JJ, Keykhah MM, Shapiro HM, et al. Cerebral hypometabolism obtained with deep pentobarbital anesthesia and hypothermia (30 C). Anesthesiology. 1978;49:159.

18. Marshall GT, James RF, Landman MP, O'Neill PJ, Cotton BA, Hansen EN, et al. Pentobarbital coma for refractory intra-cranial hypertension after severe traumatic brain injury: mortality predictions and one-year outcomes in 55 patients. J Trauma. 2010;69(2): 275-83.

19. Roberts DJ, Hall R, Kramer AH, Robertson HL, Gallagher CN, Zygun DA. Sedation for critically ill adults with severe traumatic brain injury: a systematic review of randomized controlled trials. Crit Care Med. 2011;39(12):2743-51.

20. Stocchetti N, Zanaboni C, Colombo A, Citerio G, Beretta L, Ghisoni L, et al. Refractory intracranial hypertension and "second-tier" therapies in traumatic brain injury. Intensive Care Med. 2008;34(3):461-7.

21. Wang X, Ding X, Tong Y, Zong J, Zhao X, Ren H, et al. Ketamine does not increase intracranial pressure compared with opioids: meta-analysis of randomized controlled trials. J Anesth. 2014;28(6):821-7.
22. Sahuquillo J, Martínez-Ricarte F, Poca MA. Decompressive craniectomy in traumatic brain injury after the DECRA trial. Where do we stand? Curr Opin Crit Care. 2013;19(2):101-6. This study highlights key points despite poor outcomes in DECRA trial.

23.• Flynn-O'Brien KT, Fawcett VJ, Nixon ZA, Rivara FP, Davidson $\mathrm{GH}$, Chestnut RM, et al. Temporal trends in surgical intervention for severe traumatic brain injury caused by extra-axial hemorrhage, 1995-2012. Neurosurgery. 2015;76(4):451-60. This study highlights decline in surgical intervention and reduction in inpatient mortality.

24. DuBose JJ, Barmparas G, Inaba K, Stein DM, Scalea T, Cancio LC, et al. Isolated severe traumatic brain injuries sustained during combat operations: demographics, mortality outcomes, and lessons to be learned from contrasts to civilian counterparts. J Trauma Acute Care Surg. 2011;70(1):11-8.

25. Nirula R, Millar D, Greene T, McFadden M, Shah L, Scalea TM, et al. Decompressive craniectomy or medical management for refractory intracranial hypertension: an AAST-MIT propensity score analysis. J Trauma Acute Care Surg. 2014;76(4):944-55. Highlights the role of medical management in refractory intracranial hypertension.

26. Hartings JA, Vidgeon S, Strong AJ, Zacko C, Vagal A, Andaluz N, et al. Surgical management of traumatic brain injury: a comparativeeffectiveness study of 2 centers. J Neurosurg. 2014;120(2):434-46. Demonstrated variation in surgical management between 2 Academic Medical Centers and improved outcomes with craniectomy.

27. Papazian L, Albanese J, Thirion X, Perrin G, Durbec O, Martin C. Effect of bolus doses of midazolam on intracranial pressure and cerebral perfusion pressure in patients with severe head injury. $\mathrm{Br}$ J Anesth. 1993;71(2):267-71.

28. Marmarou A, Lu J, Butcher I, McHugh GS, Mushkudiani NA, Murray GD, et al. IMPACT database of traumatic brain injury: design and description. J Neurotrauma. 2007;24:239-50.

29. Olivecrona M, Koskinen L. The IMPACT prognosis calculator used in patients with severe traumatic brain injury treated with an ICP-targeted therapy. Acta Neurochir (Wien). 2012;154(9):156773.

30. Olivecrona M, Olivecrona Z. Use of the CRASH study prognosis calculator in patients with severe traumatic brain injury treated with an intracranial pressure-targeted therapy. J Clin Neurosci. 2013;20(7):996-1001.

31. Roozenbeek B, Lingsma HF, Lecky FE, Lu J, Weir J, Butcher I, et al. Prediction of outcome after moderate and severe traumatic brain injury: external validation of the international mission on prognosis and analysis of clinical trials (IMPACT) and corticoid randomisation after significant head injury (CRASH) prognostic models. Crit Care Med. 2012;40(5):1609-17. 